\title{
Hybrid between Ontology and quantum particle swarm optimization for segmenting noisy plant disease image
}

\author{
Eman K. Elsayed ${ }^{1}$, Mohammed Aly ${ }^{2}$
}

\begin{abstract}
One of the main risks to food security is plant diseases, but because of the absence of needed infrastructure and actual noise, scientists are faced with a difficult issue. Semantic segmentation of images divides images into non-overlapped regions, with specified semantic labels allocated.

In this paper, The QPSO (quantum particle swarm optimization) algorithm has been used in segmentation of an original noisy image and Ontology has been used in classification the segmented image. Input noisy image segmentation is limited to a classification phase in which the object is transferred to Ontology.

With 49,563 images from healthy and diseased plant leaves, 12 plant species were identified and 22 diseases, the proposed method is evaluated. The method proposed produces an accuracy of 86.22 percent for a stopped test set, showing that the strategy is appropriate. EPDO (Enhance Plant Disease Ontology) is built with the web ontology language (OWL). The segmented noisy image elements are paired with EPDO with derived features that come from QPSO. Our results show that a classification based on the suggested method is better than the state-of-the-art algorithms. The proposed method also saves time and effort for removing the noise at noise level from the input image $\sigma=70$
\end{abstract}

Keywords - Image segmentation, Ontology, quantum particle swarm optimization algorithm.

\section{INTRODUCTION}

Several actors, such as plant diseases, weather change and others, are threatening food security. Plant diseases threaten world food safety and farmers who rely on safe plants to livelihood. More than 80 percent of agricultural manufacturing is produced by smallholder farmers. The reduction in output of more than 50\% [1] was caused by diseases and pests. Moreover in small farm homes, more than 50 percent of starving individuals reside. Smallholder's farmers especially susceptible to pathogenic food production disruptions.

Researchers are trying differently to avoid plant reduction caused by pests and diseases. In this paper we show the strategy by means of an Ontology using a total of 49,563 noisy images (noisy level $\sigma=30$ ) produced publicly accessible by the PlantVillage project [2] for a total of 12 plant types of 22 illnesses (or good ones).

Our research did not receive any specific funding.

Eman K. Elsayed is with Mathematics and Computer science Dept., Faculty of Science, Alazhar University (girls branch), Cairo, Egypt,

Phone: 002-01063690685; e-mail: emankaran10@azhar.edu.eg.

Mohammed Aly is with Mathematical and Computer science Dept., Faculty of Science, Zagazig University, Zagazig, Egypt (e-mail: mhmed.aly90@yahoo.com).
The last few years have seen great advancement in computer vision as well as image recognition. To determine the classifier of images for plant disease treatment, we need a big checked dataset of injured and good plants. There really is no dataset and smaller datasets are not accessible openly. To fix this issue, we have been using the PlantVillage project which includes ten of thousands of images of good and diseased crops that are publicly and readily accessible. We used 49.563 noisy photos in this paper to categorize 12 plant types that are 22 safe or injured. The efficiency of the method proposed is evaluated on the basis of the capacity of 34 possible classes to identify right crop diseases.

For higher-level regional-based feature description, nonsupervised segmentation algorithms like mean shift [3], SLIC superpixel [4], graph-based segmentation [5], [6], JSEG [7], and TurboPixel [8] were used. These unsupervised segmentation methods prefer to cover segment items with high inner contours heterogeneous parts. The segmented areas lose sementic meaning due to semantic consistency.

The objective of this paper is together the image segmentation, object detection, and semantic solutions to identify the diseases of plants. The method suggested is distinct from the previous methods in which the segmentation integration was postponed in the close-end phase, while the proposed method applied the segmentation integration in beginning.

The rest of this paper is organized accordingly. The related work is displayed in section II. Section III presents the Enhanced Plant Disease Ontology (EPDO). Section IV explains the suggested method. Section V presents the results of the test. In section VI, conclusion of this work is presented.

\section{RELATED WORKS}

Applicability of knowledge relying on image analysis for plant diseases [1], [9]-[31] has been shown by several research. But the formalized methodology to make advanced information reusable is lacking. Many variables, including air pollution and low-temperature and stressors including pests or pathogens, can cause crop diseases primarily. Infectious plant diseases are produced by the pathogens such as bacteria, viruses and insects. We are constructing a plant disease enhancement (EPDO) for the PDO [32]-[35] as guide for crop disease as portion of a major project to create Ontologies that recognize both plant type and disease. We 
have created PDO that will provide agronomists and farmers with solutions for crop disease detection and the collection of epidemiologic statistics, to integrate information associated to both plant disease as well as plant physiology, to help interpret phenotypic plant pathogens and disease procedures in attempt to influence integrated information of both fields.

The Infectious Disease Ontology plant (IDOPlant) [36] developed. IDOPlant interoperated with both the Foundry of Open Biomedical Ontologies (OBO) partners and candidates, for example the Gene Ontology (GO), the Plant Trait Ontology (TO), and the Plant Ontology (PO). Some ontologies used to define plant diseases have been described in TABLE I.

TABLE I

INDICATES SOME EXTERNAL ONTOLOGIES FOR THE DESCRIPTION OF PLANT DISEASES.

\begin{tabular}{lll}
\hline \hline ID & Ontology Name & Domain \\
\hline TO [37] & Trait Ontology & Plant traits \\
GO [38] & Gene Ontology & $\begin{array}{l}\text { Biological processes, } \\
\text { molecular function, and sub- } \\
\text { cellular components }\end{array}$ \\
PO [39] & Plant Ontology & $\begin{array}{l}\text { plant structures and } \\
\text { development stages }\end{array}$ \\
ENVO [40] & Environment Ontology & $\begin{array}{l}\text { Environment features and } \\
\text { residents }\end{array}$ \\
CHEBI [41] & $\begin{array}{l}\text { Chemical Entities of } \\
\text { Biological Interest }\end{array}$ & chemical entities \\
PATO [42] & $\begin{array}{l}\text { Phenotypic Quality } \\
\text { Ontology }\end{array}$ & phenotypic qualities \\
NCBItaxon & NCBI Taxonomy & taxonomic classification of \\
[43]-[47] & Classification & living organisms \\
GAZ [48]- & Gazetteer & geographical information \\
53] & &
\end{tabular}

The GO, which has been developed as part of the PAMGO project [54], is connected to the IDOPlant multi-organism. The IDOPlant has been developed to cover many infectious diseases of plants. There are different extensions to the IDOPlant and existing IDO. Due to the current IDO extensions, particular diseases like malaria and Brucellosis impact human and animal life [55], [56] are concentrated in particular.

The first step in [57]-[59] was presented by the PlantPathogen Interactions Ontology (PPIO), which is the axiomation of plant-pathogen interactions. The Pseudomonas syringae pv. Tomato (PsPto) has been used to improve PPIO, where the first simulation issue is PsPto disease due to the general understanding of this pathogenic method. PPIO created on PO, TO Ontology, axioms and Pathogenic Effects phrases.

In [60] PSO Quantum Theory and QPSO algorithms proposed. As the particle can exist in QPSO throughout all generations, this enhances the variety of the population. QPSO has a strong search capacity globally. The QPSO control is greater than PSO algorithm, where it has one parameter. In terms of the search capacity, QPSO is the updated and expanded version of the PSO algorithm as well as its accuracy. QPSO algorithm particles may pop up in every search space, which focuses on the delta potential. The QPSO algorithm may skip fault the standard PSO algorithm, but it cannot ensure global convergence with probability 1. The positions and speeds of the particles in quantum space can never be evaluated concurrently. In [61] the QPSO is introduced to save running time and overcome dimensionality using the cooperative technique (CQPSO). In [62] a combination of the PSO algorithm with the growing seed region (SRG) introduced. The SRG technique performs seed segmentation of the image. The PSO algorithm is used for measuring the requirements of similarity and the average region. QPSO method [63] used to encode the present location of the particle, search particles and carry out mutations, alternately, with quantum bits, quantum rotation gate and quantum not gate.

Several segmenting techniques [3]-[8], [64]-[69] are available, and it is difficult for scientists to discover an effective technique of image segmentation. Several techniques formulate object detection in order to locate notable objects in the foreground of the image, while semantic image segmentation defines each pixel with a predetermined label. Semantic image segmentation, image segmentation, and object detection seem to be three heterogeneous issues, since pixel labels profit from the segmentation and the separation of the object detector in semantic segmentation. Semantic segmentation of images combines the hardness of segmentation and object detection with the complex. These issues have been checked by different literature techniques in any situation of their dependence. This paper presents the technique for semantic image segmentation, considering and developing image segmentation and object detection at the same time. In particular, images are divided into regular items.

\section{ENHANCE PLANT DISEASES ONTOLOGY (EPDO)}

Ontology represents formally and explicitly a conceptualization for sharing [70]. The conceptualization is now a model of the world phenomenon by ideas in the summary of this concept; Ontology means sharing a consensual knowledge, agreed upon by experts, the concept types and limitations for their use are represented explicitly, and the Ontology should be machine-readable by means of formality. Ontology defines the ideas of semantics and their links. In this paper the Web Ontology Language (OWL) is employed for ontological formalism as a knowledge representation language. Where the OWL is one of the most popular ontology languages.

The next stages in the development of EPDO are proposed:

1. Detect the Ontology domain as well as the scope.

2. Consider reuse of Ontologies that exist. We looked for the corresponding Ontology in order to identify a plant domain.

3. Calculates significant terms in Ontology. The terms are applied to define the concepts and relationships that defines the domain area.

4. Describes the hierarchy of class and classes. 
5. Set the class characteristics. Fig. 1 shows the characteristics of the data type.

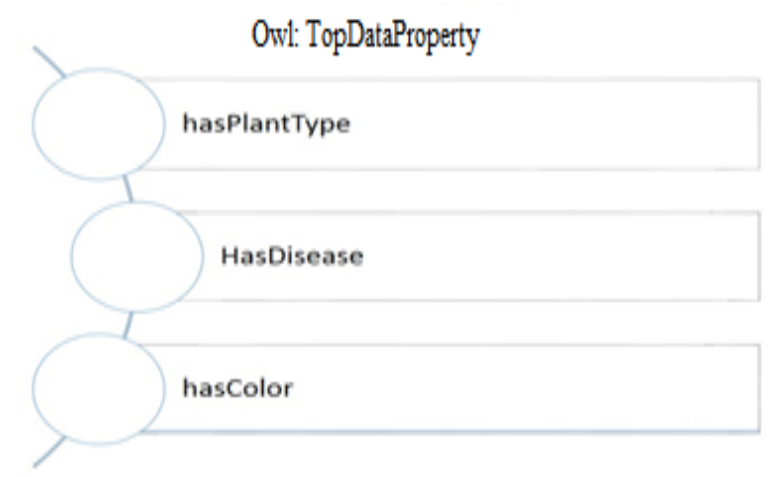

Fig. 1 Properties of data type to display the attributes extracted.

\section{Directly define the class-related constraints.}

Restrictions like domain and concepts must be defined. For illustration, the value of the hasDisease property is limited to the integer of data type.

7. Create instances. The segmentation and feature extraction module create these instances.

The PO is applied to enhance the PDO [39]. The PDO includes plant disease-specific terms and corresponding conditions imported from other ontologies, like OBI organisms; disease disorders and disease course from OGMs; ENVO habitat; GO (gene ontology) reproductive complex, and hosting (GO) reproductive complex[38]; the NCBItaxon bacterium and virus[43]- [47].

Then, Then, EPDO has created new classes, axioms, individuals, rules, and terms for the integration of the PDO. These modifications identify the leaves of plants and their diseases. Where, we added features of plant diseases, color, and various type of plant in classes and sub-classes such as 'plant disease', 'color' and 'type' sub-classes. The OWL Class Hierarchy of EPDO is presented in Fig. 2.

The EPDO varies from current PDO because the PDO concentrates on particular plant affected diseases or pathogens. By fact, 12 species of plant and 22 infectious diseases are covered by the EPDO. Additionally, as portion of the Plant Phenotype Ontology Project, the EPDO is being improved, which involves all plant stress and is not restricted to infectious diseases. Our method calls for multiple plans covering the construction of fresh terms and the importation of terms and the creation of connections with other ontologies.

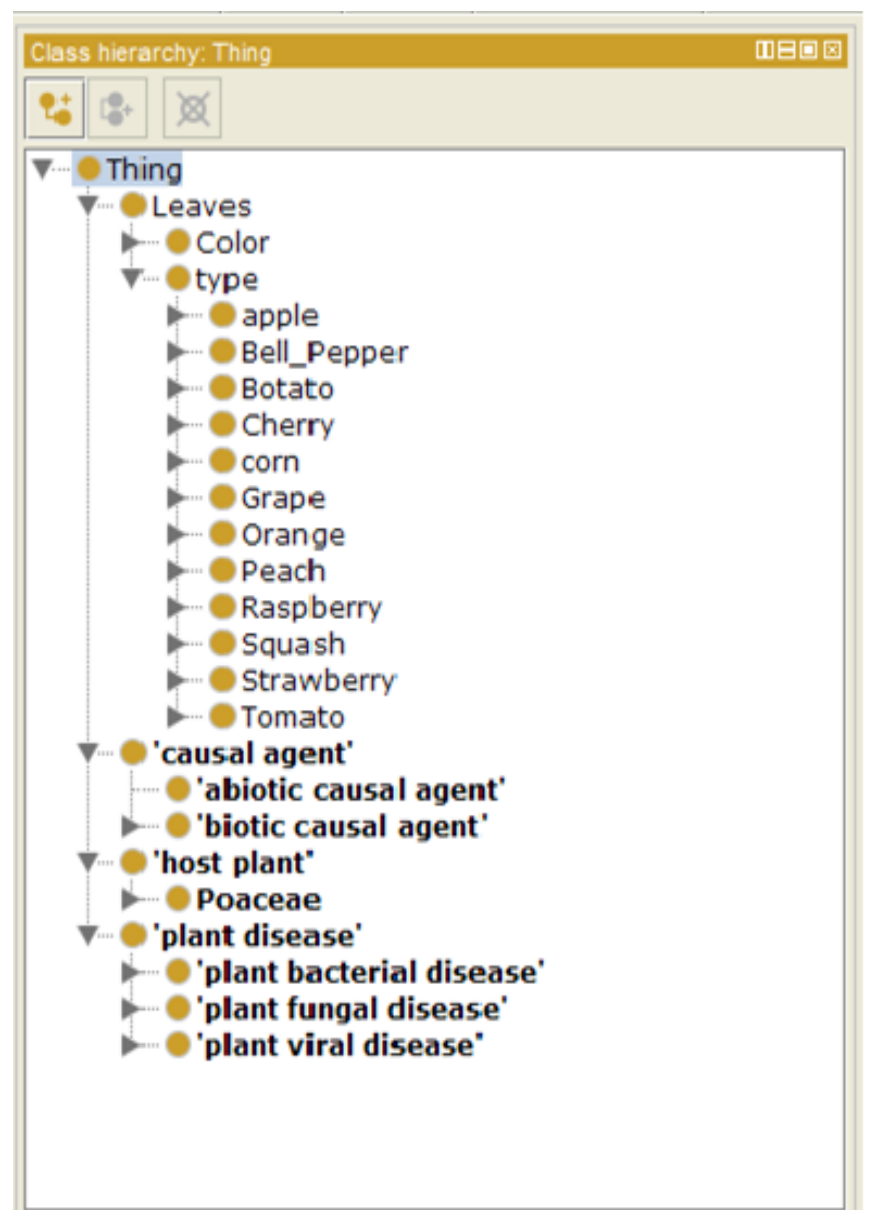

Fig. 2 Shows the OWL Class hierarchy of EPDO.

\section{A. Modularization of Ontology}

PDO is a research of pathogens (infectious organisms) and environmental (physiological) diseases of plants. Organisms like (fungi, omycetes, bacteria, viruses, and viroid), and virus like (organisms, phytoplasmas, protozoa, nematodes and parasitic plants) are all cause infectious disease. Ectoparasites including insects, mites, spinal cells or any other pests that influence the health of plants by eating plant tissues are not identified Plant pathology often includes the research on detection of pathogen, etiology of diseases, disease cycles, financial impacts, epidemiology of plant diseases, plant resistance, how plant diseases influence people and animals, pathosystem genetics and treatment of plant diseases [32].

This paper includes two components, one domain and one feature, for modularization of EPDO. The Ontology can be transferred through the modularization and skip the reuse of a whole domain of Ontology if only a part is necessary only. Relationships and appropriate concepts are only used for the modularization of Ontology in the Ontology modelling.

\section{- Ontology Domain}

The EPDO Ontology domain shows the entities which discovered the domain. In the domain, the relationship between the classes and their sub-classes are described as subset notation (드) as the following.

Leaf $s_{2}$ causal agent plant disease, host plant 5 Thing

oryza, triticum, hordeum $z e a$ 드 poaceae 
abioti viral disease, abiotic causal agent 5 causal agent $(3)$ plant bacterial disease, plant fungal disease, plant viral disease $\subseteq$ causal agent

\section{- Ontology Features}

EPDO Ontology features provide the features of various characteristics identified during the feature extraction. Eqs. 5,6 and 7 show some of the relationship between the feature classes and their sub-classes. Fig. 3 visually describes the hierarchical relation of the domain and features of EPDO Ontology.

yellow, green, black 드 color:

\section{type, color 드 leafs,}

Apple , BellPepper , Botato ,Cherry ,corn , Grape ,Orange ,

Peach , Raspberry , Squash , Strawberry $\subseteq$ type

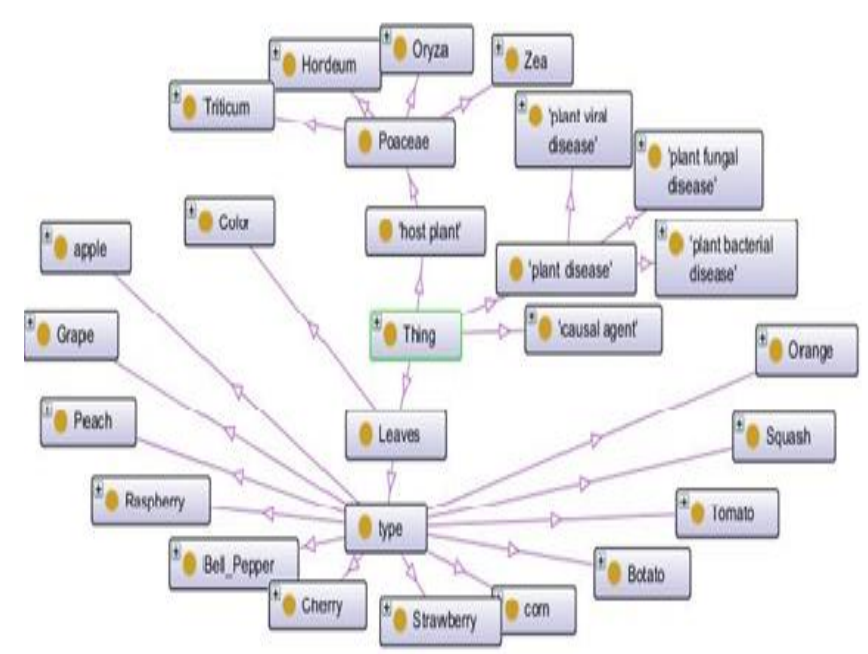

Fig. 3 The hierarchy of EPDO domain and features.

\section{PROPOSED METHOD}

The proposed method is classified into four phases. Fig. 4 illustrates the general workflow of the suggested method.

1) Segmentation and feature extraction phase.

2) connect features extraction with EPDO phase

3) Extracting threshold values phase.

4) Classification based on EPDO phase.

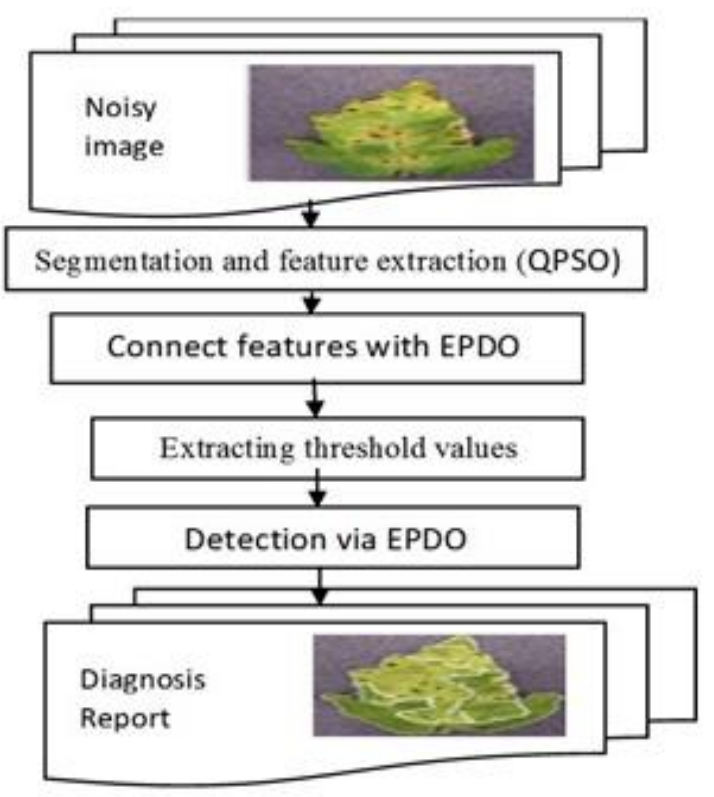

Fig.4 Proposed method phases

In the proposed method, the input is noisy image. We don't need to remove the noise from the input noisy image. So, the proposed method saves time and effort for removing the noise. Various kinds of noise may infect images like Gaussian, impulsive, speckle, or mixed noise. Image processing has several pre-processing stages. Image denoising is a major pre-processing stage in the image processing [71]-[81]. The aim of denoising images is to remove noise from the damaged images in order to assess their initial image, while preserving the appropriate characteristics and useful information of images. Due to the enhanced performance of the noisy images that are damaged by multiple noise types, several image denoising algorithms have been enhanced in recent years. In [79], [80] A wider description of the denoising methods is provided. While there are different kinds of images denoising algorithms, their efficiency in reducing noise and running time is not optimal. The input images are the same, having the same size and damaged with the additive Gaussian noise (GN) $\mathrm{N}\left(0, \sigma^{2}\right)$, where $\sigma^{2}$ is the estimated noise deviation with noise level $\sigma<=70$ (experementally). This series of tests was designed to know whether the Ontology can actually identify the characteristics of noisy test image plant diseases after we inserted the noise, or whether it's just knowing the inherent biases in the dataset.

\section{A. Segmentation and Feature Extraction phase}

The segmentation is a significant and essential step; the image is split into objects that can be defined and categorized. The outputs of the segmentation method are image objects featuring as contextual, spectral, as well as geometric. As a collection of pixels, the segmented object may have geometric attributes like shape and size. In contrast, divided objects are contextually connected by spatial relationships.

Extraction of features is the next stage in which varying numbers of features are determined for each segment of the object image. The determined feature values are transferred from Phase 1 in the proposed technique. The outcome of this stage is segmented image objects with feature values. The QPSO algorithm is applied to compute the appropriate 
parameters and then to identify the appropriate threshold values in this paper. In Ontology, the image objects, segmentation parameters and information are related to each other.

\section{A.1. Image segmentation}

\section{A.1.1. Preliminaries}

RGB has been transformed to pseudocolor (ind). As we can save a copy of the map used for that. We can convert each element of the pseudo-color map to grayscale and show it in that manner. At last, the pseudocolor map can be transformed to RGB. Without loss of information, we can not immediately transform the grayscale images back to RGB. The input noisy image transformed into pseudocolor map and then transformed the pseudocolor member into grayscale image. Let $\mathbb{L}$ is the $\left[0,1_{v} \ldots, \mathbb{L}-1\right]$ grayscale levels of an image. The grayscale levels $\mathbb{L}$ allocation is displayed in histogram $h(g)$. The histogram $h(g)$ appears as a distribution function of the probability:

$$
\begin{aligned}
& h(g)=\frac{n_{g}}{N}, h(g) \geq 0 \\
& N=\sum_{g=0}^{L-1} n_{g} \text {, and } \sum_{g=0}^{L-1} h(g)=1
\end{aligned}
$$

Where ${ }^{n_{g}}$ is described as number of pixels with gray level 9 , and $\mathbb{N}$ is the complete pixel amount in an image. A combination of the Gaussian probability function is the histogram equation:

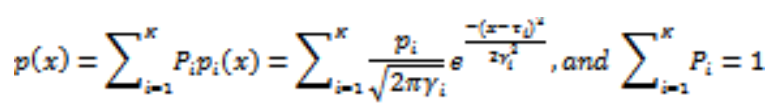

Where ${ }^{P_{\tilde{I}}}$ is a class ${ }^{\tilde{I}}$ probability, ${ }^{P_{1}(x)}$ is described in graylevel random variable ${ }^{x}$ in class $\tilde{i}_{*}$ as the probability distribution function. The mean and standard deviation of ${ }^{\text {it th }}$ the distribution probability function are $\tau_{i}$ and $V_{\mathbb{i}}$ respectively, and $\mathbb{K}$ denotes the number of classes in image. The parameters $P_{i v} \tau_{i}$ and $\gamma_{i}$ are calculated by using a mean square error (MSE) criterion. The MSE from $p\left(x_{i}\right)$ to $h\left(x_{i}\right)$ is referred to as

$$
E=\frac{1}{n} \sum_{i=1}^{n}\left[p\left(x_{i}\right)-h\left(x_{i}\right)\right]^{2}
$$

Where the histogram of n-point is considered [82].

The Eq. 9 has no recognized analytical solution but is nonlinear formula. The gradient information can be resolved via iterative approach. Where, the numerical methods approach is iterative approach based gradient data. Depending on the initialization, the ultimate solution to a gradient descent approach is hard. The QPSO will therefore be applied to evaluate the appropriate parameters and to identify the appropriate threshold values.

Although multiple segmentation [3]-[8], [64]-[69] models exist, the scientists experience a challenge of finding an effective technique of image segmentation. Despite advances in latest years there is uncertainty in the perceptual composition of image regions. In which the segmentation of images into the perceptual classification of image regions is really essential, as many apps rely on them such as scene comprehension, object detection, object identification and semantic image segmentation [70]. In the same manner as traditional methods, the suggested semantic segmentation method requires that image features have been linked by the statistical composition of the perceived setting and therefore uses clustering algorithms such as blurred c-means (FCM) as well as K-means to use separate vectors as cluster centrepieces. The features of the image will then be planned on the relevant bins. Finally, pixels form a region within the same cluster. Fig. 5 presents certain test images, which are segmented with the proposed method, and are similar to two state-of-the-art methods, JSEG [7] and SLIC [4].The first row is the initial image, The second row is an outcome of the JSEG, the third row is an outcome of the SLIC while the last line a segmented image with the proposed approach. The outcomes achieved with the proposed method are more closely related to the visual perception of humans.

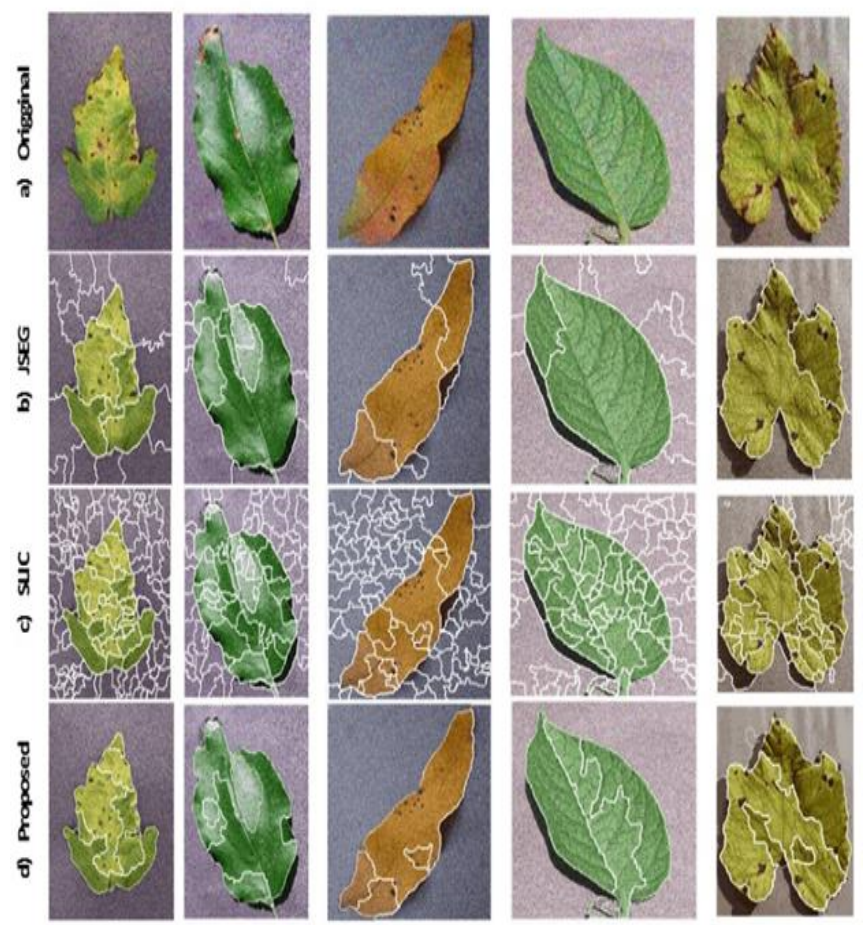

Fig. 5 illustrates examples and correlations with state-of-the-art techniques. a) Initial image, b) JSEG technique outcomes, c) SLIC technique outcomes and d) segmented images using the technique proposed.

\section{B. Extracting Threshold Value}

After using the QPSO all parameters in Eq.9 can be evaluated in the results of the appropriate threshold. The threshold results can be evaluated after measurement of the total probability error for two Gaussian neighboring functions.

$E\left(T_{i}\right)=P_{i+1} E_{1}\left(T_{i}\right)+P_{i} E_{2}\left(T_{i}\right), i=1,2,3, \ldots, K-1$

Where,

$E_{1}\left(T_{i}\right)=\int_{-m}^{T_{i}} p_{i+1}(x) d x_{x}$

$E_{2}\left(T_{i}\right)=\int_{T_{i}}^{-\infty} p_{i}(x) d x$

The probability of error in classifying pixels in class $(i+1)^{\text {th }}$ to class $\left({ }^{i}\right)^{\text {th }}$ as well as in class $\left({ }^{i}\right)^{\text {th }}$ to class $(i+1)^{\text {th }}$ are $E_{1}\left(T_{i}\right)$ and $E_{2}\left(T_{i}\right)$ respectively. $T_{i}$ is the threshold value from class $\left({ }^{i}\right)^{\text {th }}$ to class $\left({ }^{i}+1\right)$ th . After selecting $T_{\mathbb{i}}$ the $E\left(T_{i}\right)$ is minimal. The following Eq. (14) is applied to calculate the optimal ${ }^{T}$ : 
$A T_{\mathrm{i}}^{2}+B T_{\mathrm{i}}+C=0$

Where

$A=\gamma_{\mathrm{i}}^{2}-\gamma_{\mathrm{i}+1}^{2}$

$B=2\left(\tau_{i} \gamma_{i+1}^{2}-\tau_{i+1} \gamma_{i}^{2}\right)$.

$C=\left(\gamma_{i} \tau_{i+1}\right)^{2}-\left(\gamma_{i+1} \tau_{i}\right)^{2}+2\left(\gamma_{i} \gamma_{i+1}\right)^{2} \ln \left(\frac{\gamma_{i+1} P_{i}}{\gamma_{i} P_{i+1}}\right)$.

There are two potential solutions in the earlier quadratic equation; only one of them can operate.

\section{Classification and Connecting to EPDO}

Where the noisy input image is segmented, and features are extracted after phase 1 . In phase 2 , these features will compare with the features that existed in EPDO, if they are same the input noisy image will classify, and its disease, type and color will detect.

Semantic Web Rules Language (SWRL) has been applied to write the rules of ontology. These rules that applied to classify the plant diseases are focused on the SWRL. In SWRL, there are two components of the rules 1) an antecedent, 2) consequent, the two components consisting of a set of atoms. The atoms can be shaped

$C(x), P(x, y)$, sameAs $(x, y)$ differentFrom $(x, y)$, or builtin $\left(r_{v}, x_{v}\right.$ , where the OWL description is $C$, the OWL property is $P, r$ is a built-in relation, and $x$ and $y$ are two variables [83]. The consequent as well as an antecedent are written as $a_{1} a_{2} a_{n}$. The question mark $\left(e_{0} g_{x} ? x\right)$ is referred to the variables. We may use SWRL to limit the number values of the factors to the built in greaterThan Or Equal and lessThan. For instance, we have a standard for ' plant disease ' as:

Thing(? $x)$ a Leafs(? $x)^{\text {a }}$ type(? $\left.x\right)$ a apple(? $\left.x\right) \rightarrow$ healthy(? $\left.x\right)$ (16)

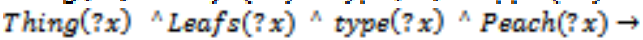

Peach Bacterial Spot, Xanthomonas campestris(? $x$ )

Thing $(? x)^{\wedge}$ Leafs $(? x)^{\wedge}$ type $(? x)^{\wedge}$ Orange $(? x) \rightarrow$ Orange Huanglongbing (Citrus Greening), Candidatus Liberibacterspp $(? x)$

These rules were applied to classify plants using Protégé 4.3.0. Each image segment was classified into each plant class. The outcome of the classification is a high level entry for segmentation and this method is referred to as EPDObased ranking.

\section{V.Experiment Results}

\section{A. Data}

Fig. 6 displays a distinct content of noisy images. For the uniformly chosen collection of leaves, Fig. 6 demonstrates distinct variants of the same leaf. No datasets are available and smaller datasets are not available for free. In attempt to fix this issue, we have been using the PlantVillage project, where tens of thousands of images of healthy as well as diseased plants are publicly and readily accessible. We used 49,563 noisy images in this paper for the classification of 12 plant species with 22 diseased or good ones. The efficiency of the proposed technique is evaluated in 29 possible categories depending on its capacity to identify the right couple crop diseases.
Dataset: the proposed method on the [2] database is being computed. The plan PlantVillage [2] offers an overview of 49, 563 images of 12 plant species (or healthy) with 22 diseases, allocated to them with a variety of 29 class labels. The class label corresponds to a couple of plant disease; it only takes the image of the plant leaf to identify the crop disease pair. The plant disease pair description from the PlantVillage dataset is displayed in Fig. 6.

Features, Baselines and Metrics: the efficiency of the method proposed is comparable with state-of - the-art classification accuracy methods. These state-of-the-art approaches include the Layered object [84], [85], Contextual cues [86], [87], Harmony [88, 89], SvrSegm [90], HIM [91], DPG model [92], [93], and Graphical model [94], [95]. In [2], the performance is computed based on the ratio between intersection of the inferred segmentation and the ground truth, and of their union as Eq. (19).
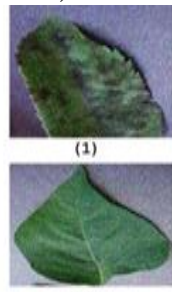

(6)

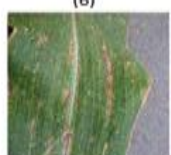

(11)

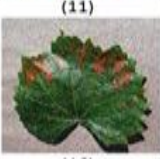

(16)

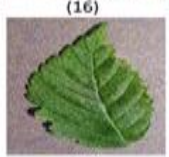

(21)

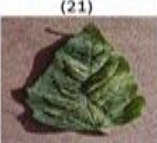

(26)

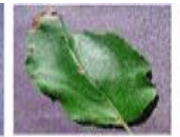

(2)

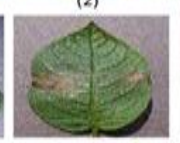

(7)
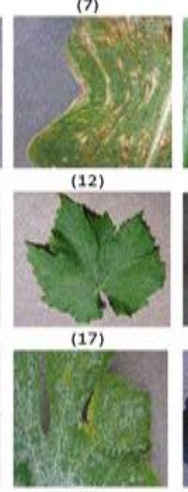

(22)

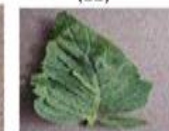

(27)
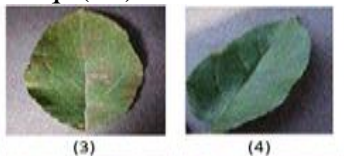

(4)

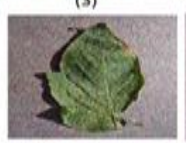

(8)
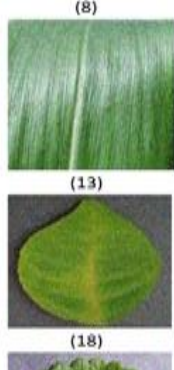

(18)

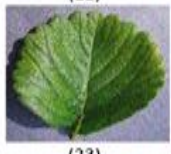

(23)

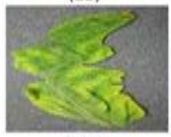

(28)

(9)

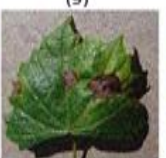

(14)

(19)

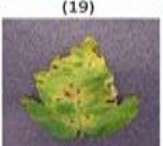

(24)

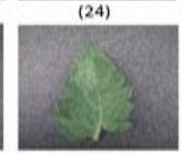

(29)

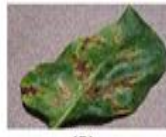

(5)
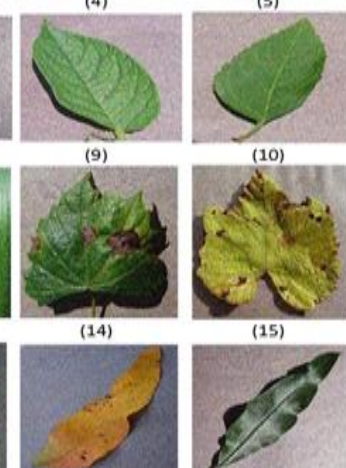

Fig. 6 Example of leaves images from the PlantVillage dataset, representing every plant-disease pair used. (1) Apple Scab, Venturia inaequalis (2) Apple Black Rot, Botryosphaeria obtusa (3) Apple Cedar Rust, Gymnosporangium juniperi-virginianae (4) Apple healthy (5) Bell Pepper Bacterial Spot, Xanthomonas campestris (6) Bell Pepper healthy (7) Potato Early Blight, Alternaria solani (8) Potato Late Blight, Phytophthora infestans (9) Potato healthy (10) Cherry healthy (11) Corn Gray Leaf Spot, Cercospora zeae-maydis (12) Corn Common Rust, Puccinia sorghi, (13) Corn healthy (14) Grape Black Rot, Guignardia bidwellii (15) Grape Leaf Blight, Pseudocercospora vitis (16) Grape Black Measles (Esca), Phaeomoniella aleophilum, Phaeomoniella chlamydospora (17) Grape Healthy (18) Orange Huanglongbing (Citrus Greening), Candidatus Liberibacter spp. (19) Peach Bacterial Spot, Xanthomonas campestris (20) Peach healthy (21) Raspberry healthy (22) Squash Powdery Mildew, Erysiphe cichoracearum (23) Strawberry Healthy (24) Tomato Bacterial Spot, Xanthomonas campestris pv. vesicatoria (25) Tomato Early Blight, Alternaria solani (26) Tomato Two Spotted Spider Mite, Tetranychus urticae (27) Tomato Mosaic Virus (28) Tomato Yellow Leaf Curl Virus (29) Tomato healthy. 


\section{B. Validation}

Comparison of the ground truth data with classification result known as validation. Total precision of classification results has been measured by method of a confusion matrix Eq. (19) [70].

total accuracy $=\frac{T P}{T P+F P+F N}$

Where TP is True Positives, FP is False Positive, and FN is False Negative pixels. By validating the classification outcomes, TP, FP, as well as FN are computed against the ground truth data.

In TABLE II, III, and IV. In TABLE II describe the outcomes of our classifications. The confusion matrix of the 5 classes is shown in TABLE II. In the confusion matrix, in the first row the labels display the classifier predictions, although in the first left column the ground-reality labels are displayed. The input ' 6 ' for instance in the third row shows that six test samples have been estimated as peach, but are indeed of the Potato class. The diagonal records display right results (the expected and the ground truth labels match). Where the right predictions are the diagonal entries.

TABLE II

SHOWS CONFUSION MATRIX FOR THE TRAINING SET.

\begin{tabular}{clllll}
\hline $\begin{array}{c}\text { Classifier } \\
\text { Ground } \\
\text { truth }\end{array}$ & Orange & Potato & Peach & Grape & Tomato \\
\hline Orange & 1674 & 19 & 7 & 4 & 2 \\
Potato & 15 & 1320 & 6 & 12 & 5 \\
Peach & 1 & 7 & 932 & 22 & 6 \\
Grape & 1 & 1 & 5 & 1064 & 8 \\
Tomato & 3 & 7 & 2 & 6 & 410 \\
\hline \hline
\end{tabular}

The efficiency measurements of the proposed technique and of the state-of -the-art techniques on the database [2] are displayed in TABLE III. In the first row of the labels the tested image appeared, while in the first left column labels the state-of - the-art algorithms are displayed. As shown in TABLE III, in the seven images of $1,2,3,5,7,8$ and 9 , the proposed technique achieves high accuracy. In addition of global precision, the approach proposed also outperforms other approaches. Contextual cues [86], [87] merging highand low-level characteristics Contextual cues and using the conditional random field (CRF) are $85 \%$ accessible. An established CRF hierarchical structure for combining contextual data on varying scales is the harmony potential technique [88], [89] that reaches 84\%.82\% and $76 \%$ respectively were the resulting of HIM [91] and Graphical model [94], [95]. Layered object's [84], [85] overall accuracy reaches $81 \%$. The SvrSegm [90] and DPG model [92], [93] are $83 \%$ and $81 \%$ respectively of the global accuracy. $88 \%$ of the proposed approach are superior to other approaches, likely because of the consistency of the semantic implementation, weighting of features and the implementation of data and knowledge required.
TABLE III

DISPLAYS OUTCOMES FOR VARIOUS APPROACHES FOR CLASSIFICATION ON [2]. THE BETTER OUTCOMES ARE MARKED BY BOLD.

\begin{tabular}{ccccccccccc}
\hline \hline Image No. & 1 & 2 & 3 & 4 & 5 & 6 & 7 & 8 & 9 & Avg. \\
\cline { 1 - 6 } $\begin{array}{c}\text { Algorithms } \\
\text { Layered }\end{array}$ & 71 & 68 & 73 & 76 & 78 & 77 & 80 & 81 & 82 & 76.2 \\
$\begin{array}{c}\text { Contextual } \\
\text { cues }\end{array}$ & 88 & 92 & 80 & 74 & 86 & 75 & 85 & 80 & 81 & 82.3 \\
Harmony & 62 & 89 & 87 & $\mathbf{8 3}$ & 81 & 83 & 84 & 83 & 85 & 81.9 \\
$\begin{array}{c}\text { SvrSegm } \\
\text { HIM }\end{array}$ & 80 & 83 & 78 & 77 & 80 & $\mathbf{8 4}$ & 82 & 82 & 84 & 81.1 \\
$\begin{array}{c}\text { DPG } \\
\text { model }\end{array}$ & 79 & 83 & 68 & 75 & 77 & 79 & 77 & 80 & 80 & 77.6 \\
$\begin{array}{c}\text { Graphical } \\
\text { model }\end{array}$ & 76 & 90 & 67 & 59 & 70 & 55 & 75 & 59 & 78 & 69.9 \\
$\begin{array}{c}\text { Proposed } \\
\text { method }\end{array}$ & $\mathbf{8 9}$ & $\mathbf{9 5}$ & $\mathbf{8 9}$ & 76 & $\mathbf{8 7}$ & 83 & $\mathbf{8 7}$ & $\mathbf{8 4}$ & $\mathbf{8 6}$ & $\mathbf{8 6 . 2}$ \\
\hline \hline
\end{tabular}

TABLE IV, provides samples of outcomes which are found using the proposed approach for the 13 plant images. In TABLE IV, the labels in the top first row display the features that identified for each test image, while the tested image names are appeared in the first left column labels. For instance, the third row represents the color, name, and disease that detected when the noisy image ' 2 ' executed in the proposed approach.

TABLE IV

SHOWS SOME PROPERTIES OF PLANT IMAGES THAT DETECTED USING THE PROPOSED METHOD.

\begin{tabular}{|c|c|c|c|}
\hline Features & \multirow{2}{*}{ Color } & \multirow{2}{*}{ Name } & \multirow{2}{*}{ Diseases } \\
\hline Image No. & & & \\
\hline 1 & $\begin{array}{l}\text { May be } \\
\text { black or } \\
\text { green }\end{array}$ & Apple Scab & Venturia inaequalis \\
\hline 2 & green & $\begin{array}{l}\text { Apple Black } \\
\text { Rot }\end{array}$ & $\begin{array}{c}\text { Botryosphaeria } \\
\text { obtusa }\end{array}$ \\
\hline 3 & green & $\begin{array}{c}\text { Apple Cedar } \\
\text { Rust }\end{array}$ & $\begin{array}{l}\text { Gymnosporangium } \\
\text { juniperi-virginianae }\end{array}$ \\
\hline 4 & green & Apple & Apple healthy \\
\hline 5 & green & $\begin{array}{c}\text { Bell Pepper } \\
\text { Bacterial } \\
\text { Spot }\end{array}$ & $\begin{array}{l}\text { Xanthomonas } \\
\text { campestris }\end{array}$ \\
\hline 6 & green & Bell Pepper & Bell Pepper healthy \\
\hline 7 & green & $\begin{array}{l}\text { Potato Early } \\
\text { Blight }\end{array}$ & Alternaria solani \\
\hline 8 & green & $\begin{array}{l}\text { Potato Late } \\
\text { Blight }\end{array}$ & $\begin{array}{l}\text { Phytophthora } \\
\text { infestans }\end{array}$ \\
\hline 9 & green & Potato & Potato healthy \\
\hline 10 & green & Cherry & Cherry healthy \\
\hline 11 & Green & $\begin{array}{l}\text { Corn Gray } \\
\text { Leaf Spot }\end{array}$ & $\begin{array}{c}\text { Cercospora zeae- } \\
\text { maydis }\end{array}$ \\
\hline 12 & $\begin{array}{l}\text { May be } \\
\text { green or } \\
\text { yellow }\end{array}$ & $\begin{array}{l}\text { Corn } \\
\text { Common } \\
\text { Rust }\end{array}$ & Puccinia sorghi \\
\hline 13 & green & Corn & Corn healthy \\
\hline
\end{tabular}

\section{CONCLUSION}

As per the essential of quick treatment of plant diseases from corrupted images. This paper proposed approach for 
semantic image segmentation which used Ontology and Quantum particle Swarm Optimization. The approach proposed needs Ontology for the domain of interests to be constructed. Domain knowledge of plant characteristics can be extracted from Ontology. We use the PDO Ontology to improve our method and to perform all Ontologies required for logical descriptions by using existing terms. In this paper, EPDO has been produced, in part, thought merging terms and axioms from PDO with new classes, axioms, individuals, rules, and terms that describe plants and its diseases.

This research helps to enhance the use of Ontological techniques for the detection and development of plant diseases. It offer a new approach, which is used Ontology classification rules, to immediately extract threshold values. The current outcomes indicate that the approach proposed is stronger than the state-of-the-art approaches. The proposed approach will also save time and effort needed to clear the noise from input image until noise level $\sigma=70$.

\section{ACKNOWLEDGMENT}

The authors thank the editors and the anonymous reviewers for their valuable suggestions.

\section{REFERENCES}

[1] Mohanty, P. Sharada, David P. Hughes, and Marcel Salathé, "Using deep learning for image-based plant disease detection," Frontiers in plant science, vol. 7, pp.1419, 2016.

[2] PlantVillage. (Accessed on 25 Jan 2019). Available: https://www.plantvillage,org/en/plant images.

[3] Comaniciu, Dorin, and Peter Meer, "Mean shift: A robust approach toward feature space analysis," IEEE Transactions on Pattern Analysis \& Machine Intelligence, vol. 5, pp. 603-619, 2002.

[4] R. Achanta, A. Shaji, K. Smith, A. Lucchi, P. Fua, and S. Süsstrunk, "SLIC superpixels compared to state-of-the-art superpixel methods," IEEE transactions on pattern analysis and machine intelligence, vol. 34, no.11, pp. 2274-2282, 2012.

[5] Li, Zhenguo, Xiao-Ming Wu, and Shih-Fu Chang, "Segmentation using superpixels: A bipartite graph partitioning approach," 2012 IEEE Conference on Computer Vision and Pattern Recognition. IEEE, 2012.

[6] P. F. Felzenszwalb, and D. P. Huttenlocher, "Efficient graph-based image segmentation," International journal of computer vision, vol. 59, no. 2, pp. 167-181, 2004

[7] Deng, Yining, and B. S. Manjunath, "Unsupervised segmentation of color-texture regions in images and video," IEEE transactions on pattern analysis and machine intelligence, vol. 23, no. 8, pp. 800-810, 2001.

[8] A. Levinshtein, A. Stere, K. N. Kutulakos, D. J.Fleet, S. J. Dickinson, and K. Siddiqi, "Turbopixels: Fast superpixels using geometric flows," IEEE transactions on pattern analysis and machine intelligence, vol. 31, no. 12, pp. 2290-2297, 2009.

[9] S. Sankaran, A. Mishra, R. Ehsani and C. Davis, "A review of advanced techniques for detecting plant diseases," Computers and Electronics in Agriculture, vol. 72, no. 1, pp. 1-13, 2010.

[10] Barbedo and Jayme Garcia Arnal, "Digital image processing techniques for detecting, quantifying and classifying plant diseases," SpringerPlus, vol. 2, no. 1, pp. 660, 2013.

[11] Mutka, M. Andrew, and Rebecca S. Bart, "Image-based phenotyping of plant disease symptoms," Frontiers in plant science, vol. 5, pp. 734, 2015.

[12] Mahlein and Anne-Katrin, "Plant disease detection by imaging sensors-parallels and specific demands for precision agriculture and plant phenotyping," Plant disease, vol. 100, no. 2, pp. 241-251, 2016.

[13] C. H. Bock, G. H. Poole, P. E. Parker and T. R. Gottwald, "Plant disease severity estimated visually by digital photography and image analysis, and by hyperspectral imaging," Critical Reviews in Plant Sciences, vol. 29, no. 2, pp. 59-107, 2010.

[14] Khirade, D. Sachin and A. B. Patil, "Plant disease detection using image processing," 2015 International conference on computing communication control and automation. IEEE, 2015.
[15] Mutka, M. Andrew, and S. Bart. Rebecca, "Image-based phenotyping of plant disease symptoms," Frontiers in plant science, vol. 5, pp. 734, 2015.

[16] Lowe, Amy, Nicola Harrison, and Andrew P. French, "Hyperspectral image analysis techniques for the detection and classification of the early onset of plant disease and stress," Plant methods, vol. 13, no. 1, pp. 80 , 2017.

[17] Steinmetz, F. Nicole, Michael Bruckman, and Lauren Randolph, "Coated plant virus imaging agents," U.S. Patent Application, No. 10/207,014.

[18] Hamilton, Robert G, “Assessment of human allergic diseases," Clinical Immunology, Content Repository Only, pp.1283-1295, 2019

[19] K. Golhani, S. K. Balasundram, G. Vadamalai and B. Pradhan, "A review of neural networks in plant disease detection using hyperspectral data,'Information Processing in Agriculture, vol. 5, no. 3, pp. 354-371, 2018

[20] J. D. Pujari, R. S. Yakkundimath, S. Jahagirdar and A. M. Byadgi, "Quantitative detection of soybean rust using image processing techniques," Journal of Crop Protection, vol. 5, no. 1, pp. 75-87. 2016.

[21] Greenberg and Bernard, "Flies and disease: II. Biology and disease transmission," Princeton University Press, vol. 5363, 2019.

[22] A. J. Mastin, F. van den Bosch, F. van den Berg and S. R. Parnell, "Quantifying the hidden costs of imperfect detection for early detection surveillance," Philosophical Transactions of the Royal Society, vol. B 374, no. $1776,2019$.

[23] Pantazi, Xanthoula Eirini, Dimitrios Moshou, and Alexandra A. Tamouridou, "Automated leaf disease detection in different crop species through image features analysis and One Class Classifiers," Computers and electronics in agriculture, vol. 156, pp. 96-104, 2019.

[24] J. Abdulridha, R. Ehsani, A. Abd-Elrahman and Y. Ampatzidis, "A remote sensing technique for detecting laurel wilt disease in avocado in presence of other biotic and abiotic stresses," Computers and Electronics in Agriculture, vol.156, pp. 549-557, 2019.

[25] Tsuchiya and Shinsuke, "Leaked gas detection device and leaked gas detection method," U.S. Patent Application, no. 10/190,975, 2019.

[26] N. A. Wulff, C. G. Fassini, V. V. Marques, E. C. Martins, D. A B.Coletti, D. D. C. Teixeira, ... and J. M. Bové, "Molecular characterization and detection of 16 SrIII group phytoplasma associated with Huanglongbing symptoms," Phytopathology, vol.109, no. 3, pp. 366374, 2019.

[27] E. H. Achbani, H. Mazouz, A. Benbouazza and S. Sadik, "First detection of Pantoea ananatis, the causal agent of bacterial center rot of onion in Morocco,"Journal of Crop Protection, vol. 5, no.1, pp. 11-17, 2015 .

[28] M. A. Gomez, Z. D. Lin, T. Moll, R. D. Chauhan, L. Hayden, K. Renninger and R. S. Bart, " Simultaneous CRISPR/Cas9-mediated editing of cassava eIF $4 \mathrm{E}$ isoforms $\mathrm{nCBP}-1$ and $\mathrm{nCBP}-2$ reduces cassava brown streak disease symptom severity and incidence," Plant biotechnology journal, vol.17, no.2, pp. 421-434, 2019.

[29] R. M. Sadek, S. A. Mohammed, A. R. K. Abunbehan, A. K. H. A. Ghattas, M. R. Badawi, M. N. Mortaja, ... and S. S. Abu-Naser, "Parkinson's Disease Prediction Using Artificial Neural Network," International Journal of Academic Health and Medical Research (IJAHMR), vol. 3, no. 1, pp. 1-8, 2019

[30] De Visser, Jan, "Peronospora resistance in spinacia sp," U.S. Patent Application, no. 10/226,014, 2019.

[31] Gupta, C. Ramesh, "Biomarkers in toxicology,"Academic Press, 2019.

[32] Plant Ontology PO. (Accessed on 6 Feb 2019). Available: http://wiki.plantOntology.org/index.php/Plant Disease Ontology.

[33] De Oliveira, Christelle and Audrey Moutat, "From Controversy to Media Controversy: Analysis of Communication Strategies Concerning the Health Risk of Growing Limousin Apples," Food and Health: Actor Strategies in Information and Communication, vol. 2, pp. 29-51, 2019.

[34] M. A. Haendel, J. A. McMurry, R. Relevo, C. J. Mungall, P. N. Robinson and C. G. Chute, "A census of disease ontologies," Annual Review of Biomedical Data Science, vol.1, pp. 305-331, 2018.

[35] E. Cerruti, C. Comino, A. Acquadro, G. Marconi, A. M. Repetto, A B. Pisanu, ... and E. Portis, "Analysis of DNA Methylation Patterns Associated with In Vitro Propagated Globe Artichoke Plants Using an EpiRADseq-Based Approach," Genes, vol. 10, no. 4, pp. 263, 2019.

[36] R. Walls, B. Smith, E. Justin, G. Albert and W. S. Dennis, "A plant disease extension of the Infectious Disease Ontology," 2012.

[37] S. M.Edwards, I. F. Sørensen, P. Sarup, T. F. Mackay and P. Sørensen, "Genomic prediction for quantitative traits is improved by mapping variants to gene ontology categories in Drosophila melanogaster," Genetics, vol. 203, no. 4, pp. 1871-1883, 2016. 
[38] Fruzangohar, Mario, Esmaeil Ebrahimie and David L. Adelson, "A novel hypothesis-unbiased method for Gene Ontology enrichment based on transcriptome data," PloS one, vol. 12, no. 2, 2017.

[39] Smaili, Z. Fatima, Xin Gao and Robert Hoehndorf, "Formal axioms in biomedical ontologies improve analysis and interpretation of associated data," 2019

[40] A. R. Hardisty, W. K. Michener, D. Agosti, E. A. García, L. Bastin, L. Belbin and R. De Giovanni, "The Bari Manifesto: An interoperability framework for essential biodiversity variables," Ecological Informatics, vol. 49, pp. 22-31, 2019.

[41] N. Swainston, J. Hastings, A. Dekker, V. Muthukrishnan, J. May, C. Steinbeck and P. Mendes, "libChEBI: an API for accessing the ChEBI database," Journal of cheminformatics, vol. 8, no.1, pp. 11, 2016.

[42] S. Sarntivijai, Y. Lin, Z. Xiang, T. F. Meehan, A. D. Diehl, U. D. Vempati, ... and Y. Liu, "CLO: the cell line ontology," Journal of biomedical semantics, vol. 5, no.1, pp.37, 2014.

[43] Kahlke, Tim and Peter J. Ralph, "BASTA-Taxonomic classification of sequences and sequence bins using last common ancestor estimations," Methods in Ecology and Evolution, vol.10, no.1, pp. 100103, 2019

[44] Xue, Xingsi, Zhi Hang and Zhengyi Tang, "Interactive biomedical ontology matching," PloS one, vol. 14, no. 4, 2019.

[45] C. Loeffler, A. Karlsberg, E. Eskin, D. Koslicki and S. Mangul, "Analysis of multiple fungal sequence repositories highlights shortcomings in microbial databases," bioRxiv, pp. 497867, 2019.

[46] Mohan, Sunil and Donghui Li, "Med Mentions: A Large Biomedical Corpus Annotated with UMLS Concepts," arXiv preprint arXiv, vol.1902, no. 09476, 2019.

[47] L. M. Schriml, E. Mitraka, J. Munro, B. Tauber, M. Schor, L. Nickle, ... and K. Bisordi, "Human Disease Ontology 2018 update: classification, content and workflow expansion," Nucleic acids research, vol. 47, no. D1, pp. D955-D962, 2018.

[48] B. J. Stucky, J. P. Balhoff, N. Barve, V. Barve, L. Brenskelle, M. H. Brush, ... and A. Lucky, "Developing a vocabulary and ontology for modeling insect natural history data: example data, use cases, and competency questions," Biodiversity data journal, vol. 7, 2019.

[49] Koester, Christopher, "Redefining Blight in Arizona's Government Property Lease Excise Tax (GPLET) Abatement," Ariz. St. LJ, vol. 50, pp. 1319, 2018.

[50] Leane, Elizabeth, Ben Maddison and Kimberley Norris, "Beyond the Heroic Stereotype: Sidney Jeffryes and the Mythologising of Australian Antarctic History," 2019.

[51] Oudah, Mai and Khaled Shaalan, "NERA 2.0: Improving coverage and performance of rule-based named entity recognition for Arabic," Natural Language Engineering, vol. 23, no. 3, pp. 441-472, 2017.

[52] Garg, Sanjay, "Studies in Indo-Muslim History by SH Hodivala Volume I: A Critical Commentary on Elliot and Dowson's History of India as Told by Its Own Historians (Vols. I-IV) \& Yule and Burnell's HobsonJobson," Routledge, 2018.

[53] Pandya, Shubhada, "An outlier in public health history in India: ATW Simeons's scheme for rural medical relief, Kolhapur, 1943-47," The National medical journal of India, vol. 30, no.2, pp.103, 2017.

[54] M. G. Giglio, C. W. Collmer, J. Lomax and A. Ireland, "Applying the Gene Ontology in microbial annotation," Trends in microbiology, vol. 17, no.7, pp. 262-268, 2009.

[55] P. Topalis, E. Mitraka, I. Bujila, E. Deligianni, E. Dialynas, I. SidenKiamos and C. Louis, "IDOMAL: an ontology for malaria," Malaria journal, vol. 9, no.1, pp. 230, 2010.

[56] Lin, Yu, Zuoshuang Xiang, and Yongqun He, "Brucellosis Ontology (IDOBRU) as an extension of the Infectious Disease Ontology," Journal of biomedical semantics, vol. 2, no. 1, pp. 9, 2011.

[57] A. R. Iglesias, M. E. Aranguren, A. R. González and M. D. Wilkinson, "Plant Pathogen Interactions Ontology (PPIO)," IWBBIO, 2013.

[58] W. Song, N. Qi, C. Liang, F. Duan and H. Zhao, "Soybean root transcriptome profiling reveals a nonhost resistant response during Heterodera glycines infection," PloS one, vol. 14, no. 5, 2019.

[59] X. Zheng, X. Li, B. Wang, D. Cheng, Y. Li, W. Li, ... and A. P. Macho , "A systematic screen of conserved Ralstonia solanacearum effectors reveals the role of RipAB, a nuclear-localized effector that suppresses immune responses in potato," Molecular plant pathology, vol. 20, no. 4, pp. 547-561, 2019.

[60] Sun, Jun, Wenbo Xu and Bin Feng, "A global search strategy of quantum-behaved particle swarm optimization," IEEE Conference on Cybernetics and Intelligent Systems, Vol. 1, 2004.

[61] H. Gao, W. Xu, J. Sun and Y. Tang, "Multilevel thresholding for image segmentation through an improved quantum-behaved particle swarm algorithm," IEEE transactions on instrumentation and measurement, vol. 59, no. 4, pp. 934-946, 2009.

[62] F. Mohsen, M. M. Hadhoud, K. Moustafa and K. Ameen ,"A new image segmentation method based on particle swarm optimization," Int. Arab J. Inf. Technol., vol. 9, no. 5, pp. 487-493, 2012.

[63] Huang, Yourui and Shuang Wang, "Multilevel thresholding methods for image segmentation with Otsu based on QPSO," 2008 Congress on Image and Signal Processing, vol. 3, 2008.

[64] B. Fernando, E. Fromont, D. Muselet and M. Sebban, "Discriminative feature fusion for image classification," 2012 IEEE Conference on Computer Vision and Pattern Recognition, 2012.

[65] Wu, Lei, Steven CH Hoi and Nenghai Yu, "Semantics-preserving bag of-words models and applications," IEEE Transactions on Image Processing, vol. 19, no. 7, pp. 1908-1920, 2010.

[66] M. Xian, Y. Zhang, H. D. Cheng, F. Xu, B. Zhang and J. Ding, "Automatic breast ultrasound image segmentation: A survey," Pattern Recognition, vol. 79, pp. 340-355, 2018.

[67] Wu, Zifeng, Chunhua Shen and Anton Van Den Hengel, "Wider or deeper: Revisiting the resnet model for visual recognition," Pattern Recognition, vol. 90, pp. 119-133, 2019.

[68] S. H. E. N. Xiaohui, S. Cohen, P. Wang, B. Russell, B. Price and J. EISENMANN, "U.S. Patent Application," no. 10/290,112, 2019.

[69] G. S. Fu, Y. Levin-Schwartz, Q. H. Lin and D. Zhang, "Machine Learning for Medical Imaging," Journal of Healthcare Engineering, 2019. [70] M. Zand, S. Doraisamy, A. A. Halin and M. R. Mustaffa, "Ontologybased semantic image segmentation using mixture models and multiple CRFs," IEEE Transactions on Image Processing, vol. 25, no. 7, 32333248, 2016.

[71] Hu, Haijuan, Jacques Froment and Quansheng Liu, "A note on patchbased low-rank minimization for fast image denoising," Journal of Visual Communication and Image Representation, vol. 50, pp. 100-110, 2018.

[72] X. Li, H. He, R. Wang and J. Cheng, "Superpixel-guided nonlocal means for image denoising and super-resolution," Signal Processing, vol. 124, pp. 173-183, 2016.

[73] J. Liu, Y. Wang, K. Su and W. He, "Image denoising with multidirectional shrinkage in directionlet domain," Signal Processing, vol. 125 , pp. 64-78, 2016.

[74] Zhong, Hua, Ke Ma and Yang Zhou, "Modified BM3D algorithm for image denoising using nonlocal centralization prior," Signal Processing, vol. 106, pp. 342-347, 2015.

[75] Afonso, V. Manya, and João MR Sanches, “A total variation recursive space-variant filter for image denoising," Digital Signal Processing, vol. 40, pp. 101-116, 2015.

[76] S. Oh, H. Woo, S. Yun and M. Kang, "Non-convex hybrid total variation for image denoising," Journal of Visual Communication and Image Representation, vol. 24, no. 3, pp. 332-344, 2013.

[77] Y. Wu, B. Tracey, P. Natarajan and J. P. Noonan, "James-Stein type center pixel weights for non-local means image denoising," IEEE Signal Processing Letters, vol. 20, no. 4, pp. 411-414, 2013.

[78] W. Zhou, M. Fei, H. Zhou and K. Li, "A sparse representation based fast detection method for surface defect detection of bottle caps," Neurocomputing, vol. 123, pp. 406-414, 2014.

[79] D. H. Shin, R. H. Park, S. Yang and J. H. Jung, "Block-based noise estimation using adaptive Gaussian filtering," IEEE Transactions on Consumer Electronics, vol. 51, no.1, pp. 218-226, 2005.

[80] Shang, Li, "Non-negative sparse coding shrinkage for image denoising using normal inverse Gaussian density model," Image and Vision Computing, vol. 26 no. 8, pp.1137-1147, 2008.

[81] Jain, Paras and Vipin Tyagi, "A survey of edge-preserving image denoising methods," Information Systems Frontiers, vol. 18, no.1, pp. 159$170,2016$.

[82] Gonzalez, Rafael C., and Richard E. Woods, "Digital Image Processing Addison-Wesley," Reading, Ma 2, 1992.

[83] S. Rajbhandari, J. Aryal, J. Osborn, R. Musk and A. Lucieer, "Benchmarking the applicability of ontology in geographic object-based image analysis," ISPRS International Journal of Geo-Information, vol. 6, no. 12, pp. 386, 2017. 
[84] Y. Yang, S. Hallman, D. Ramanan and C. C. Fowlkes, "Layered object models for image segmentation," IEEE Transactions on Pattern Analysis and Machine Intelligence, vol. 34, no. 9, pp. 1731-1743, 2011.

[85] B. Zhou, H. Zhao, X. Puig, T. Xiao, S. Fidler, A. Barriuso and A. Torralba, "Semantic understanding of scenes through the ade20k dataset," International Journal of Computer Vision, vol. 127, no. 3, pp. 302-321, 2019.

[86] Zhou, Chongbo, and Chuancai Liu, "Semantic image segmentation using low-level features and contextual cues," Computers \& Electrical Engineering, vol. 40, no. 3, pp. 844-857, 2014.

[87] Q. Zhou, W. Yang, G. Gao, W. Ou, H. Lu, J. Chen and L. J. Latecki, "Multi-scale deep context convolutional neural networks for semantic segmentation," World Wide Web, vol. 22, no. 2, pp. 555-570, 2019.

[88] J. M. Gonfaus, X. Boix, J. Van de Weijer, A. D. Bagdanov, J. Serrat and J. Gonzalez, "Harmony potentials for joint classification and segmentation," 2010 IEEE computer society conference on computer vision and pattern recognition, 2010.

[89] Haridoss, Rekha and Samundiswary Punniyakodi, "Compression and Enhancement of Medical Images Using Opposition Based Harmony Search Algorithm," Journal of Information Processing Systems, vol. 15, no. 2, 2019.

[90] Carreira, João, Fuxin Li and Cristian Sminchisescu, "Object recognition by sequential figure-ground ranking," International journal of computer vision, vol. 98, no. 3, pp. 243-262, 2012.

[91] L. Zhu, Y. Chen, Y. Lin, C. Lin and A. Yuille, "Recursive segmentation and recognition templates for image parsing," IEEE Transactions on Pattern Analysis and Machine Intelligence, vol. 34, no. 2 , pp. 359-371, 2011.

[92] A. Lucchi, Y. Li, X. Boix, K. Smith and P. Fua, "Are spatial and global constraints really necessary for segmentation?," 2011 International Conference on Computer Vision IEEE, 2011.

[93] L. Xiao, J. Wang, X. Qiu, Z. Rong and X. Zou, "Dynamic-SLAM: Semantic monocular visual localization and mapping based on deep learning in dynamic environment," Robotics and Autonomous Systems, vol. 117, pp. 1-16, 2019.

[94] Zhang, Lei and Qiang Ji, "Image segmentation with a unified graphical model," IEEE Transactions on Pattern Analysis and Machine Intelligence, vol. 32, no. 8, 1406-1425, 2009.

[95] Q. Zhou, W. Yang, G. Gao, W. Ou, H. Lu, J. Chen and L. J. Latecki, "Multi-scale deep context convolutional neural networks for semantic segmentation," World Wide Web, vol. 22, no. 2, pp. 555-570, 2019.

Eman K. Elsayed received the Ph.D in computer science in 2005 at Alazhar University, and received the master of computer science at Cairo University in 1999, received the bachelor of science, mathematics and computer science at Department of Cairo University in 1994. She published 32 papers until 2016 in data mining, ontology engineering, e-learning,image processing and software engineering. Also she published 2 books in formal methods and event B on Amazon database. She is a member in egyptian mathematical society and intelligent computer and information systems society. Mathematics and Computer science Department, Faculty of science, Alazhar University, Cairo, Egypt. Corresponding author. Email: emankaran10@azhar.edu.eg. Postal code 11668. Tele: 00201063690685.

Mohammed Aly received the master of computer science at Zagazig University in 2017, received the Bachelor of Science, mathematics and computer science at Department of Zagazig University in 2012. He published many papers in image processing such as medical imaging and image processing. His research interests include multimedia content analysis, indexing and retrieval, pattern recognition, and machine learning. Mathematical and Computer science Dept., Faculty of Science, Zagazig University, Zagazig, Egypt. Email: mhmed.aly90@yahoo.com. Postal code 11668. Tele: 00201204096617
Creative Commons Attribution License 4.0 (Attribution 4.0 International, CC BY 4.0)

This article is published under the terms of the Creative Commons Attribution License 4.0

https://creativecommons.org/licenses/by/4.0/deed.en_US 